$\Rightarrow$ GENETICS

\section{GWAS identifies 24 new loci for renal function}

A meta-analysis of genome-wide association studies (GWAS) has identified 24 novel genetic loci that are associated with renal function (based on estimated glomerular filtration rate), and has confirmed 29 previously identified loci. "The majority of the genes that we identified at these loci were predominantly expressed in renal tissues, suggesting that the genetic determinants of renal function might be largely mediated through direct effects within the kidney, and not as a result of indirect extra-renal effects that might cause kidney damage," says researcher Cristian Pattaro.

The initial analysis included $>130,000$ individuals of European ancestry, and was subsequently replicated in $>$ 42,000 individuals. The identified loci were then corroborated in individuals of African American and East Asian descent. Characterization of the loci by complementary bioinformatic and functional approaches found that many of the identified gene products were enriched in pathways involved in renal development, renal structure, and transmembrane transporter activity, as well as in glucose metabolism and homeostasis. Furthermore, the identified genetic variants showed preferential mapping to gene regulatory elements in kidney-specific genes.

"Our analyses are currently being extended to incorporate more genetic variants that can be inferred with improved imputation, in order to better refine the causal genes and variants in the associated regions, and to uncover novel loci that were missed by lack of genotyping," explains Pattaro. "In the future, we will expand the range of kidney phenotypes to better characterise renal function, include more diverse populations, and increase the age range of the study participants to help us differentiate between genes that are required for renal development and genes that might have a major role in ageing."

\section{Jessica K. Edwards}

ORIGINAL ARTICLE Pattaro, C. et al. Genetic associations at 53 loci highlight cell types and biological pathways relevant for

kidney function. Nat. Commun. http://www.nature.com/

ncomms/2016/160121/ncomms10023/full/ncomms10023.htm 